\title{
Sehnenrupturen am Fuß
}

\author{
Jens Goronzy, Stefan Rammelt
}

\section{Zusammenfassung}

Weniger als $10 \%$ der Sehnenverletzungen am menschlichen Körper treten am Fuß und am oberen Sprunggelenk auf. Rupturen können degenerativ oder traumatisch bedingt sein, treten aber nur selten aufgrund eines akuten Unfalls auf, dann zumeist als direkte Durchtrennung an einem scharfen Gegenstand. Die Therapie muss der Ätiologie der Sehnenruptur und der Funktion der Sehne individuell angepasst werden. Akute Rupturen werden zumeist direkt genäht, degenerative Veränderungen müssen $z$.T. speziell mit anderen Sehnen rekonstruiert werden. Nur bei begleitenden Fehlstellungen oder Arthrosen sind Osteotomien oder Arthrodesen erforderlich.

\section{Tendon Ruptures at the Foot}

Less than $10 \%$ of tendon injuries occur in the foot and ankle. Only a small proportion is caused by acute trauma, mostly through direct laceration. The majority of ruptures, however, are caused by degeneration or attrition. Treatment has to be adjusted to the cause of tendon ruptures. Acute traumatic injuries are treated with a direct tendon suture. Chronic and degenerative ruptures have to be addressed individually. Depending on the deformity and functional deficit, different treatment options include tendon reconstruction, tendon transfer, corrective osteotomy, or arthrodesis.

\section{Einleitung}

Sehnen übertragen die im Muskel entwickelte Kraft auf einen definierten Punkt jenseits eines Gelenks und führen somit zur aktiven Gelenkbewegung. Das Sehnengewebe ist durch die wellenförmige Konfiguration der Kollagenfasern um maximal 3\% dehnbar und aufgrund der scherenförmigen Anordnung der Verbindungsfasern um weitere $5 \%$ effektiv verlängerbar (Abb.1). Da die Spannungs-Dehnungs-Kurve von Sehnen nicht linear ist, kommt es ab einer Dehnung von 6-10\% zur Ruptur. Die mittlere Reißfestigkeit von Sehnen wird im statischen Versuch mit $4,67 \mathrm{kp} / \mathrm{mm}^{2}$ und im dynamischen Versuch mit $10,1 \mathrm{kp} / \mathrm{mm}^{2}$ angegeben [1]. $\mathrm{Zu}$ beachten ist jedoch, dass durch Reduktion der Zellzahl und des Zellgehalts sowie Abnahme der Fa-

OP-JOURNAL 2015; 31: 193-199

(c) Georg Thieme Verlag KG Stuttgart · New York DOI http://dx.doi.org/10.1055/s-0041-109444 serdichte eine altersabhängige Abnahme der Reißfestigkeit besteht.

\section{Epidemiologie}

Die langen Sehnen des Fußes können 4 verschiedenen Muskelgruppen zugeordnet werden. Die häufigste Ruptur ereignet sich hierbei an der Achillessehne. In der europäischen Bevölkerung betrifft dies 6-18 Personen pro 100000 Einwohner, v.a. bei sportlichen Aktivitäten. Wir möchten in der folgenden Arbeit jedoch über die Sehnenverletzungen der restlichen 3 Muskelgruppen, die prozentual seltener auftreten und teilweise auch erst verspätet erkannt werden, referieren. In einer Analyse von 1014 Sehnenrupturen an der Mayo Klinik zwischen 1945 und 1954 zeigten sich 14,1\% an der unteren Extremität, davon allein 4,2\% an der Achillessehne und dem M. triceps surae und nur 3,8\% an den übrigen extrinsischen und intrinsischen Sehnen des Fußes [2].

\section{Pathomechanik}

Sehnenrupturen können prinzipiell durch 5 verschiedene Pathomechanismen entstehen [3]:

- direktes Trauma mit einer offenen, scharfen Durchtrennung der Sehne

- indirektes Trauma mit einer plötzlichen, unerwarteten Überdehnung und akuter Sehnenruptur

- Sehnenlazerationen bei Frakturen an scharfen Knochenkanten oder durch Interposition bei Luxationen bzw. Luxationsfrakturen

- repetitives Trauma, welches durch Ermüdung und Überlastung zur Sehnenruptur führen kann

- degenerative Rupturen haben als Ursache z. B. die entzündliche bzw. rheumatoide Arthritis, Hyperurikämie, Diabetes mellitus, lokale und systemische Steroide und können auch idiopathisch auftreten

Eine genaue Unterscheidung, ob es sich um eine traumatische oder eine degenerative Sehnenverletzung handelt, ist z.T. nur schwer $z u$ treffen. In vielen Fällen besteht eine Kombination von verschiedenen Pathomechanismen, wie z.B. bei einem Volleyballspieler, der im Vorfeld während des Trainings mehrere repetitive Traumata erlitten hat und dem während des Turniers bei der plötzlichen Überdehnung des Unterschenkels die Achillessehne reißt. Hier ist davon auszugehen, dass die akute traumatische Belastung eine geringere sein muss, dem Patienten ist jedoch nur der plötzliche Sehnenriss bewusst.

\section{Direkte offene Sehnendurchtrennungen}

Die direkte offene Sehnendurchtrennung am Fuß (direktes Trauma) ist wesentlich seltener als Sehnenverletzungen durch ein indirektes oder repetitives Trauma und kann nicht mit der Anzahl von direkten Sehnenverletzungen an der Hand verglichen werden. Die Beugesehnenverletzungen werden zumeist durch einen Tritt in eine Glasscherbe 
oder einen anderen scharfen Gegenstand verursacht. Verletzungen an den Strecksehnen werden hingegen durch fallende Objekte (Küchenmesser), Fahrradspeichen oder $z$.B. Rasenmäher verursacht.

Gerade bei kleineren Schnittwunden werden Sehnenverletzungen nicht selten im Rahmen der Erstversorgung übersehen [3]. Die klinische Untersuchung mit der Detektion von Funktionsausfällen distal der Wunde ist zur Beurteilung wegweisend.

Zur besseren Beurteilung der Funktionsübertragung der Sehne bzw. zur Diagnostik einer möglichen Retraktion der Sehnenstümpfe kann die Sonografie eingesetzt werden. In jedem Fall besteht die Indikation zur Exploration der Wunde mit ggf. Erweiterung des Zugangs zur Lokalisation der Sehnenstümpfe. Dieses Vorgehen sollte unter Anlage einer Blutsperre erfolgen. Bezüglich der End-zuEnd-Sehnennaht existieren mehrere Techniken, die propagiert werden, wobei die Abb. 2 einen Überblick über die gebräuchlichen Verfahren gibt.

\section{Sehnen und Muskeln der tiefen Beugerloge}

\section{Tibialis-posterior-Sehne}

Anatomie

Der vom N. tibialis innervierte M. tibialis posterior hat seinen Ursprung im Bereich der Membrana interossea cruris mit den benachbarten Arealen der Fibula und Tibia. Er verläuft mit seiner Sehne in einem Sulcus am Hinterrand des Innenknöchels und inseriert mit 5 Armen an der Tuberositas ossis navicularis, am plantaren Os cuneiforme mediale und laterale sowie an den Basen der Ossa metatarsalia II-IV. Dynamisch gesehen ist der Muskel v.a. ein wichtiger Supinator. Pathophysiologisch geht die Dysfunktion der Tibialis-posterior-Sehne mit einem Pes planovalgus (Knick-Plattfuß) einher. Die komplette traumatische Ruptur der Tibialis-posterior-Sehne kommt sehr selten vor und ist bis auf einzelne Fälle mit einer Lazeration an einer Fraktur des Innenknöchels assoziiert [4]. Inkomplette traumatische Rupturen bzw. Elongationen nach starken Pronationsverletzungen oder Frakturen werden teilweise erst spät mit der Entwicklung eines Pes planovalgus entdeckt. Chronisch degenerative Rupturen entstehen zumeist beim weiblichen Geschlecht im

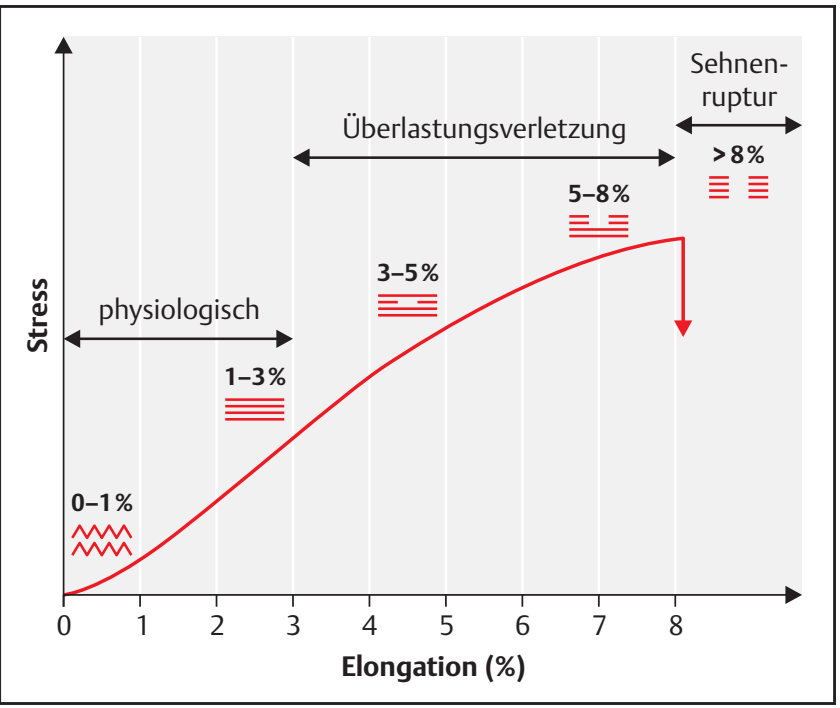

Abb. 1 Schematische Darstellung der Dehnbarkeit einer gesunden Sehne nach Maffulli [10].

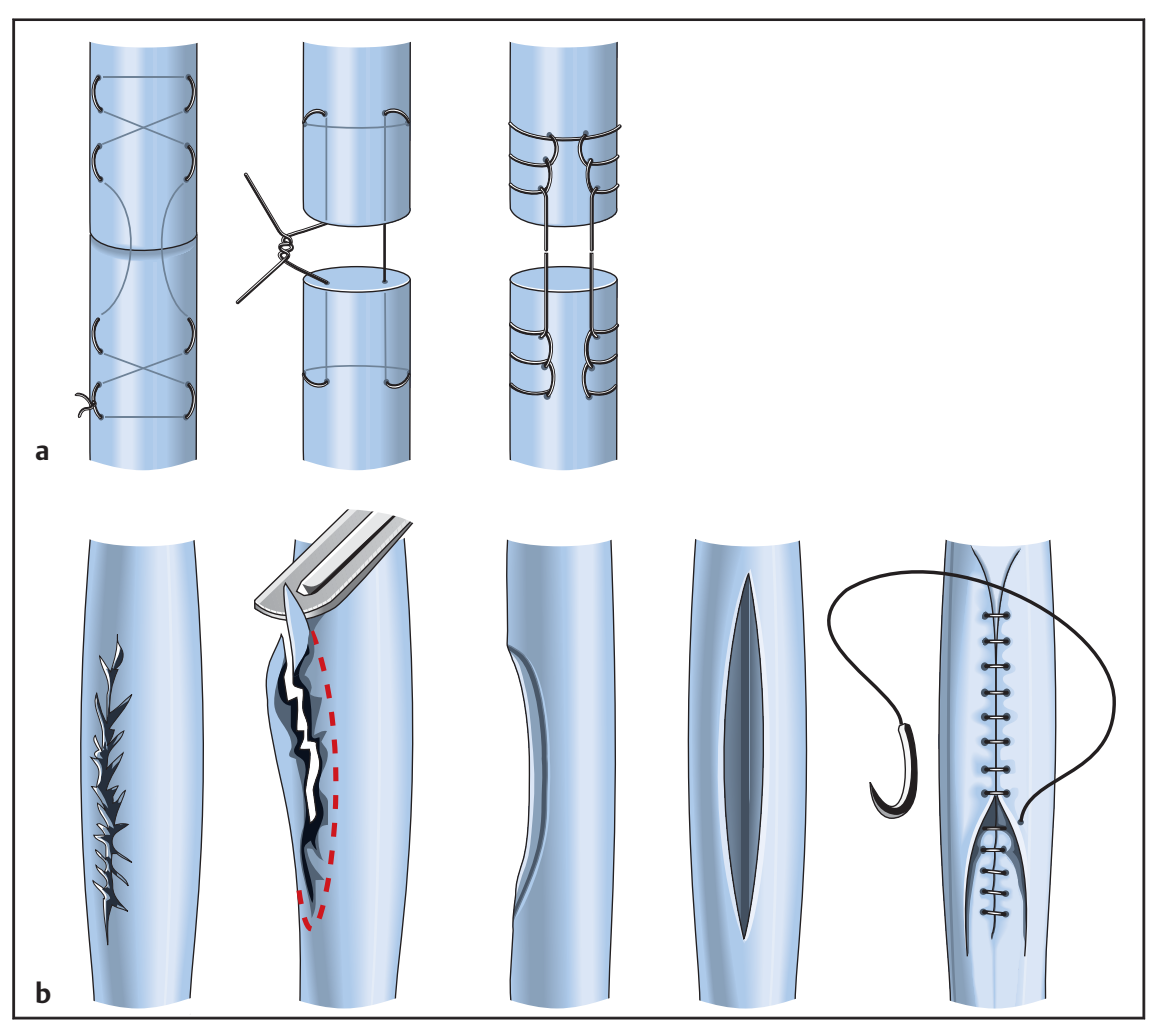

Abb. 2a und b Sehnennahttechniken. a Direkte Sehnennaht bei akuter Ruptur nach Kirchmayr/Kessler, Bunnell bzw. Krackow. b Débridement und fortlaufende Naht bei chronischer Längsruptur bzw. Auffaserung.

6. und 7. Dezennium. Inflammatorische Prozesse wie chronische Tenosynovitiden, seronegative Spondylopathien, Diabetes mellitus wie auch Alter und Übergewicht, die zur Dehnung und Degeneration der Sehne führen, beeinflussen die Sehnenfunktion erheblich. Zumeist reißt die Sehne im Bereich eines hypovaskulären Areals direkt hinter und unter dem Innenknöchel.

\section{Diagnostik}

Zur Beurteilung der akuten kompletten Ruptur der Tibialis-posterior-Sehne ist die klinische Untersuchung aufgrund der Überlagerung der Beschwerden bei Innenknöchelfraktur oder lateraler subtalarer Luxation oft nicht wegweisend. Intraoperativ sollte bei Repositionshindernissen die Darstellung der Sehne erfolgen. Einrisse des Retinakulums sowie 
Einblutungen und Mikrorupturen als Zeichen einer Elongation müssen im Operationsbericht zur Beurteilung von Folgeschäden vermerkt werden.

Patienten mit einer degenerativen Bandruptur haben zumeist einen Druckschmerz mit Schwellung und Überwärmung der Weichteile hinter dem Innenknöchel und weiter distal im Sehnenverlauf. Gleichzeitig entstehen Schmerzen bei der verstärkten Supination und Plantarflexion gegen Widerstand. Klinisch weist das „too many toes sign“ auf einen Rückfußvalgus, indem vermehrt die lateralen Zehen sichtbar werden, wenn der Untersucher gerade hinter dem Patienten steht. Der Zehenspitzenstand informiert den Arzt über die Rigidität der Deformierung. Korrigiert sich die Rückfußachse vollständig oder zum Teil, kann noch eine gelenkerhaltende Operation durchgeführt werden. Ist der Rückfuß bereits rigide, kommt meist nur noch eine korrigierende Arthrodese infrage. Die Abflachung des medialen Fußgewölbes gibt erste Hinweise auf einen Pes planovalgus. Eine Diagnosesicherung der Tibialis-posterior-Sehnenruptur kann durch MRT (Sensitivität 95\%, Spezifität 100\%) oder durch Sonografie, hier jedoch nur für vollständige Rupturen, mit gleicher Genauigkeit durchgeführt werden. Zusätzlich werden das Ausmaß knöcherner Fehlstellung oder bereits bestehende Arthrosen im Röntgen mit Belastungsaufnahmen von beiden Füßen in 2 Ebenen sowie des oberen Sprunggelenks in 2 Ebenen festgestellt werden.

Im klinischen Alltag wird die chronische Tibialis-posterior-Sehneninsuffizienz nach Johnson und Strom in die Stadien I-III und modifiziert nach Myerson und Corrigan zusätzlich in Stadium IV unterteilt (Tab. 1) [5,6].

\section{Therapie}

Bei der vollständigen traumatischen Sehnenruptur erfolgt nach der Versorgung der knöchernen Verletzung die primäre Sehnennaht. Wir präferieren hierzu nach Glättung der Sehnenstümpfe eine Sehnennaht nach Kirchmayr und Kessler mit einer doppelt armierten PDS-Naht mit der Stärke 2.0. Inkomplette traumatische Rupturen sollten im Unterschenkel-Cast für 5-6 Wochen ruhiggestellt werden, um eine schleichende komplette Ruptur oder rezidivierende posttraumatische Tendovaginitis zu verhindern.

Tab. 1 Einteilung der klinischen Stadien der Tibialis-posterior-Dysfunktion nach Johnson und Strom [5]

\begin{tabular}{llll}
$\begin{array}{l}\text { Sta- } \\
\text { dium }\end{array}$ & Klinik & $\begin{array}{l}\text { Sehnen- } \\
\text { status }\end{array}$ & $\begin{array}{l}\text { radiologischer } \\
\text { Befund }\end{array}$ \\
\hline I & $\begin{array}{l}\text { Schmerzen, Schwellung hinter dem Innen- } \\
\text { knöchel, verstärkt bei Supination, Einbein- } \\
\text { zehenstand }\end{array}$ & $\begin{array}{l}\text { Tenosyno- } \\
\text { vitis }\end{array}$ & keine Deformität \\
& $\begin{array}{l}\text { flexibler Pes planovalgus } \\
\text { Ila (kompensiert): eingeschränkter Einbein- } \\
\text { zehenstand } \\
\text { II (dekompensiert): kein Einbeinzehenstand }\end{array}$ & $\begin{array}{l}\text { Elongation, } \\
\text { Degenera- } \\
\text { tion }\end{array}$ & $\begin{array}{l}\text { Subluxation im } \\
\text { Talonavikular- } \\
\text { gelenk }\end{array}$ \\
& $\begin{array}{l}\text { rigider Pes planovalgus, Vorfußvarus > 10 } \\
\text { subfibulares Impingement, Ruheschmerz } \\
\text { (lateral > medial) }\end{array}$ & Ruptur & $\begin{array}{l}\text { Arthrosen im } \\
\text { Tripel-Gelenk- } \\
\text { komplex }\end{array}$ \\
\hline III & $\begin{array}{l}\text { Valgusfehlstellung im oberen Sprunggelenk } \\
\text { mit Schmerzen über dem lateralen Gelenk- } \\
\text { aspekt, Überdehnung des Lig. deltoideum }\end{array}$ & Ruptur & $\begin{array}{l}\text { Valgusarthrose im } \\
\text { oberen Sprung- } \\
\text { gelenk }\end{array}$ \\
\hline IV & & &
\end{tabular}

Die Therapie der degenerativen Sehnenrupturen erfolgt in Abhängigkeit vom Stadium der Sehnendegeneration, dem Ausmaß der Beschwerden und der vorliegenden Fehlstellung entsprechend dem Stadium nach Johnson und Strom (s. Tab. 1). Die Literatur hierzu ist jedoch sehr kontrovers, sodass wir im folgenden Verlauf unseren eigenen Ansatz erläutern werden.

Im Stadium I empfehlen wir noch das konservative Vorgehen. Es sollte die Verordnung von festen Einlagen zur aktiven Anregung der Fußbinnenmuskulatur und passiven Unterstützung des medialen Fußgewölbes sowie Fußgymnastik und Spiraldynamik erfolgen. Nur bei anhaltenden Beschwerden, insbesondere beim Sportler, ist eine Tenosynovektomie mit Transfer der Flexor-digitorum-longusSehne indiziert.

Im Stadium II erfolgt eine modifizierte Evans-Osteotomie zur Verlängerung der lateralen Fußsäule. Zur Ausschaltung der pathologischen Lasteinleitung ist meist eine Verlängerung des Gastrocnemius-Sehnenspiegels nach Strayer („Gastroc Slide“) bei isolierter Verkürzung des M. gastrocnemicus erforderlich. Bei instabiler medialer Fußsäule mit hypermobilem 1. Strahl sollte die gleichzeitige navikulokuneiforme sowie tarsometatarsale Arthrodese des 1. Strahles erfolgen. Bei ausgeprägtem Rückfußvalgus erfolgt zusätzlich eine medialisierende Kalkaneusosteotomie.

Im Stadium III erfolgt die reorientierende Triple-Arthrodese (Arthrodese des Talonavikular-, Kalkaneokuboid- und des Subtalargelenks) zumeist in Kombi- nation mit einem Gastroc Slide. Falls das Kalkaneokuboidgelenk nicht arthrotisch verändert ist, beschränkt sich die Arthrodese auf das Talonavikular- und Subtalargelenk zum Erhalt einer gewissen Flexibilität der lateralen Fußsäule.

Bei Stadium IV ist zusätzlich zur Korrektur des Rückfußes auf die Korrektur des oberen Sprunggelenks zu achten. So kann bei noch nicht vorhandenen oder nur mäßigen Arthrosezeichen die Korrektur der Deformität im Rückfuß mit einer supramalleolaren Korrekturosteotomie und Rekonstruktion des medialen Bandapparats oder bei fortgeschrittenen Arthrosezeichen mit der Implantation einer Sprunggelenkendoprothese verbunden werden.

\section{Nachbehandlung}

Die Nachbehandlung einer vollständigen Sehnenruptur erfolgt im Cast in Neutralstellung des Fußes für 6 Wochen. Nach Korrekturarthrodese sollte eine Immobilisation für mindestens 6 Wochen bei $20 \mathrm{~kg}$ Teilbelastung im UnterschenkelCast erfolgen. Bei verminderter Knochenqualität oder Knochenspaninterposition kann sich die Zeit auf bis zu 12 Wochen verlängern.

\section{Flexor-hallucis-longus-Sehne}

\section{Anatomie}

Der M. flexor hallucis longus ist der kräftigste Muskel der tiefen Beugerloge und hat seinen Ursprung am distalen Drittel der Fibula sowie der Membrana interossea cruris und wird ebenfalls durch den N. tibialis innerviert. Seine Sehne 
zieht in einer dorsalen Rinne des Talus in unmittelbarer Beziehung zum Tuberculum mediale des Processus posterior tali weiter nach distal unterhalb des Sustentaculum tali des Kalkaneus, um anschließend die Sehne des M. flexor digitorum longus im Chiasma tendinum (Henry's knot) zu kreuzen und sich durch feine Sehnenfasern mit dieser zu verbinden. Der Ansatz erfolgt an der Basis der Endphalanx der Großzehe. Der Muskel unterstützt neben der Beugung im Grundgelenk auch die Plantarflexion und Supination des Fußes.

Die häufigste Verletzung der Sehne tritt durch offene Verletzung mit einem scharfen Gegenstand, wie z.B. den Tritt in eine Glasscherbe, auf. Geschlossene Rupturen treten nur selten auf und wenn, dann zumeist posttraumatisch verursacht durch überstehende Schrauben nach Versorgung einer Kalkaneusfraktur oder durch Inkarzeration zwischen Fragmenten des Sustentaculum tali des Kalkaneus.

Degenerative Veränderungen und insbesondere Spontanrupturen sind äußerst selten und sind zumeist im Bereich des oberen Flexorenretinakulums lokalisiert. Sie treten v.a. bei Extremsportlern und Balletttänzern durch eine unphysiologisch hohe Belastung auf.

\section{Diagnostik}

Die klinische Untersuchung ist bez. der Lokalisation der Sehnenruptur zielführend. Bei Verletzung der Sehne zwischen Ansatz und dem Chiasma tendinum kann eine aktive Beugung im Interphalangealgelenk nicht mehr durchgeführt werden. Ist die Beugung im Großzehengrundgelenk ebenfalls nicht mehr möglich, sind eine oder beide Sehnen des M. flexor hallucis verletzt. Bei Rupturen proximal des Chiasma tendinum besteht eine Restbeugefähigkeit durch die oben beschriebene Interaktion mit der Sehne des M. flexor digitorum longus. Dieses erschwert z.T. die Diagnosestellung. Schmerzen im Bereich des Tarsaltunnels bei der aktiven Bewegung der Zehe weisen auf eine chronische Reizung im Sinne einer Tendovaginitis stenosans hin.

Als additive Diagnostik kann bei akuten Verletzungen der Ultraschall und bei chronischen Verletzungen zusätzlich das MRT zur Beurteilung der Sehne hinzugezogen werden. Knöcherne Veränderungen bzw. die Beurteilung der Schraubenlage können am besten im CT beurteilt werden.
Therapie

Die offene Sehnendurchtrennung sollte, wie oben beschrieben, durch eine Wundexploration mit direkter Sehnennaht behandelt werden. Sehnenausrisse können über Nahtanker refixiert werden. Die initiale Therapie der Tendovaginitis stenosans sollte konservativ erfolgen. Hierzu gehören eine Sportkarenz für 3-6 Wochen mit Gabe von NSAR und physiotherapeutischen Maßnahmen. Eine operative Therapie kommt vor allem bei Leistungsportlern mit über 3 Monate anhaltenden Beschwerden unter konservativer Therapie infrage. Bei partiellen Rupturen erfolgen eine vollständige Spaltung des fibroossären Tunnels sowie das Débridement der Sehne und die fortlaufende Naht mit feinem Nahtmaterial. Operationen zur reinen Verbesserung der Beweglichkeit ohne bestehende Beschwerden sollten kritisch hinterfragt werden, da der weitgehende Verlust der Beugung im Interphalangealgelenk im Alltag keine wesentlichen funktionellen Einschränkungen bedeutet.

\section{Flexor-digitorum-longus-Sehne}

\section{Anatomie}

Der M. flexor digitorum longus hat seinen Ursprung an der mittleren Hinterseite der Tibia und wird ebenfalls vom N. tibialis innerviert. Die Sehne überkreuzt am distalen Unterschenkel die Sehne des M. tibialis posterior (Chiasma tendinum crurale), zieht gemeinsam mit der Sehne des M. tibialis posterior hinter dem Innenknöchel entlang, kreuzt, wie bereits oben beschrieben, in der medialen Planta pedis die Sehne des Flexor hallucis longus und spaltet sich weiter distal in 4 Sehnenzügel, die an der Basis des jeweiligen Zehenendglieds inserieren. Verletzungen treten typischerweise, ähnlich wie beim M. flexor hallucis longus, durch Trittverletzungen in einen scharfen Gegenstand auf. Isolierte degenerative Rupturen sind in der Literatur nicht beschrieben.

\section{Diagnostik und Therapie}

Die klinische Beurteilung erfolgt durch aktive Plantarflexion im distalen Interphalangealgelenk gegen Widerstand. Sollte die Bewegung auch im proximalen Gelenk gestört sein, ist vermutlich auch die Flexor-digitorum-brevis-Sehne durchtrennt. In beiden Fällen sollte eine Exploration der Wunde mit direkter Sehnennaht erfolgen. Bei Rupturen der Seh- ne proximal des Chiasma tendinum bleibt eine Restfunktion erhalten, weswegen keine Naht erfolgen muss. Postoperativ sollten die Patienten mit Sehnennaht für 6 Wochen im Gips in Funktionsstellung ruhiggestellt werden.

\section{Sehnen und Muskeln der Streckerloge}

\section{Tibialis-anterior-Sehne}

\section{Anatomie}

Der Muskel entspringt an den lateralen oberen 2 Dritteln der Tibia sowie der Membrana interossea cruris und wird durch den N. peroneus profundus innerviert. Seine flache Sehne läuft durch das mediale Fach des oberen Extensorenretinakulums (Lig. transversum) sowie unter 2 Schenkeln des unteren Extensorenretinakulums (Lig. cruciatum) und inseriert an der plantaren Fläche des Os cuneiforme mediale und des Os metatarsale I. Dem M. tibialis anterior werden ca. 80\% der Extensionskraft des Fußes zugeschrieben, gleichzeitig wirkt er auch supinierend.

Traumatische Rupturen entstehen zumeist durch Tibia- bzw. Pilonfrakturen oder Verletzungen an scharfen Gegenständen [3]. Degenerative Spontanrupturen treten vor allem bei Männern im 6. und 7. Lebensjahrzehnt auf und sind auf eine hypovaskuläre Zone der Sehne $3 \mathrm{~cm}$ proximal ihres Ansatzes auf Höhe des Extensorenretinakulums lokalisiert. Zumeist bestehen prädisponierende Faktoren wie Diabetes mellitus, Gicht, rheumatoide Arthritis, lokale Steroidinjektionen oder Exostosen an der Tibiavorderkante.

\section{Diagnostik}

Die Dorsalextension im Sprunggelenk nach Sehnenruptur ist eingeschränkt und sollte im Seitenvergleich getestet werden. Ein Hinweis auf eine Verletzung der M.-tibialis-anterior-Sehne ist das Einkrallen der Zehen bei Dorsalextension als Hinweis auf ein Recruitment der Zehenstrecker. Zumeist bestehen eine Schwellung über dem anteromedialen Aspekt des Sprunggelenks sowie eine tastbare Lücke im Bereich des Sehnenverlaufs. Die initialen Beschwerden sind häufig schnell rückläufig, weshalb die Diagnose häufig erst verspätet gestellt wird. Sonografie und MRT können zur Diagnosesicherung verwendet werden (Abb. 3). 

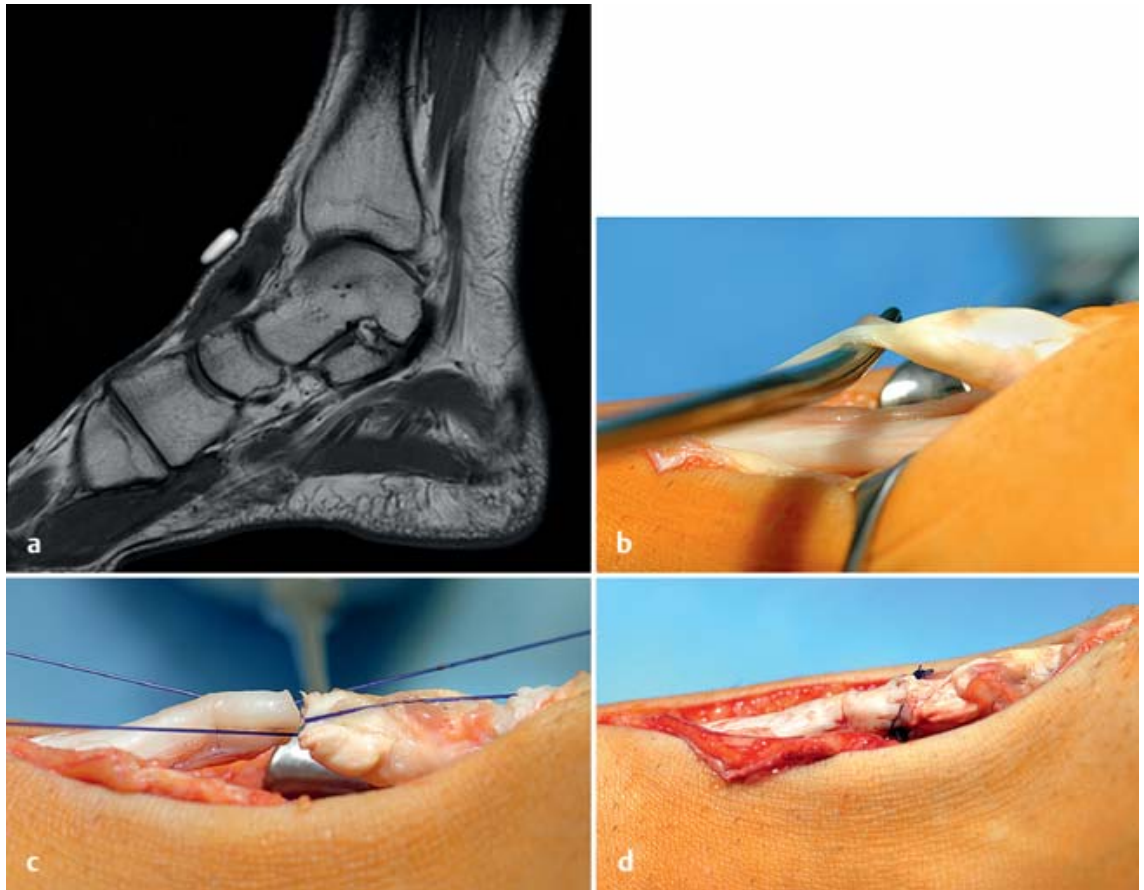

Abb. $\mathbf{3 a}$ bis $\mathbf{d}$ Verspätet diagnostizierte traumatische Ruptur der Tibialis-anterior-Sehne bei einem 41-jährigen Freizeitfußballer nach direktem Anprall. b Die Rupturstelle liegt typischerweise direkt unterhalb des oberen Strecksehnenretinakulums, die Sehnenstümpfe weisen noch Adhäsionen auf. c Nach Débridement der Sehnenenden ließen sich diese spannungsfrei adaptieren. d Daraufhin erfolgt die sekundäre Naht in der Technik von Kirchmayr/Kessler.

\section{Therapie}

Bei der akuten Ruptur besteht die Indikation zur direkten End-zu-End-Naht. Teilweise muss ein 2. proximaler Zugang zur Bergung des vorderen Sehnenstumpfs erfolgen. Zur Spaltung und anschließenden unkomplizierten Naht des Retinakulums bietet sich eine Z-förmige Spaltung an. Knöcherne Ausrisse können mit einem Nahtanker refixiert werden.

Bei verspätet diagnostizierten Sehnenrupturen sind die Therapieempfehlungen uneinheitlich. Oft ist eine direkte End-zu-End-Naht der Sehne aufgrund der Retraktion des Muskels nicht mehr möglich. Folgende Techniken werden beschrieben:

- extraanatomische Refixation (z.B. Os naviculare) [7]

- frei gedoppeltes Plantaristransplantat

- Transfer der halben oder gesamten M.-extensor-hallucis-longus-Sehne auf den distalen Stumpf der M.-tibialisanterior-Sehne sowie Transfer der M.-extensor-hallucis-brevis-Sehne auf den distalen Stumpf der M.-extensorhallucis-longus-Sehne [8]

- Transfer der M.-peroneus-tertius-Sehne

- freier Transfer der halben M.-peroneus-brevis- oder -longus-Sehne [9]
- proximal gestielte Umkippplastik der M.-tibialis-anterior-Sehne

Begleitend sollte bei einer Dorsalextension von $5^{\circ}$ bei gestrecktem Knie und positivem Silfverskiöld-Test ein Gastroc Slide erfolgen. Bei fehlender Funktion des M. tibialis anterior (z.B. Lähmungsspitzfuß, Z.n. Kompartmentsyndrom) stellt eine Rekonstruktion keine sinnvolle Therapie dar. Stattdessen muss ein Transfer, z. B. der Tibialis-posterior-Sehne, erwogen werden. Postoperativ wird der Fuß für 6-8 Wochen in einem Unterschenkel-Cast mit beschwerdeadaptierter Belastung ruhiggestellt. Anschließend können unter noch bestehender Protektion bis zur 12. Woche Bewegungsübungen begonnen werden.

\section{Extensor-hallucis-longus-Sehne}

\section{Anatomie}

Der Muskel entspringt am mittleren Drittel der medialen Seite der Fibula sowie der Membrana interossea cruris und wird vom N. peroneus profundus innerviert. Die Sehne verläuft in einem separaten Strecksehnenfach zwischen der Sehne des M. tibialis anterior (medial) und des M. extensor digitorum longus und überkreuzt auf Höhe des oberen Sprunggelenks das Gefäß-Nerven-Bün- del. Seinen Ansatz hat der Muskel an der streckseitigen Basis der Großzehenendphalanx, wobei auf Höhe der Grundphalanx die Sehnen des M. abductor und M. adductor hallucis einstrahlen.

Verletzungen der M.-extensor-hallucislongus-Sehne werden vornehmlich durch offene direkte Verletzungen durch herabfallende Gegenstände, wie z.B. Glas oder Messer oder im Rahmen von Komplextraumata, verursacht. Häufig besteht aufgrund der anatomischen Nähe eine Begleitverletzung der A. tibialis anterior bzw. A. dorsalis pedis und des N. peroneus profundus. Geschlossene Rupturen treten sehr selten auf und können durch Überlastung (Sport, hochschaftiges Schuhwerk) oder nach Eingriffen nahe der Strecksehne entstehen.

\section{Diagnostik und Therapie}

Eine aktive Streckung der Großzehe nach Sehnenverletzungen ist nicht möglich. Hinweisend ist auch der „Push-up-Test“, bei dem es bei Druck von plantar auf das Metatarsophalangealgelenk zu einer reflektorischen Beugung im Interphalangealgelenk aufgrund der Sehnendurchtrennung kommt. Eine fehlende subkutane Tastbarkeit bei Anspannung der Sehne ist ebenso evident. Bei offenen Verletzungen im OSG und Fußbereich sollte eine Verletzung der Arterie sowie des Nervs, welche durch Hypästhesie im 1. Zehenzwischenraum auffällt, ausgeschlossen werden.

Bei akuten Verletzungen sollte nach Wundexploration die direkte Sehnennaht erfolgen. Bei akuter Verletzung mit bereits bestehender Retraktion des proximalen Sehnenstumpfs kann dieser durch manuellen Druck auf die Sehnenscheide oder eine Sehnenfasszange ohne 2. Zugang geborgen werden. Bei Defektstrecken können z. B. die Extensor-hallucis-brevis-, die halbe oder vollständige benachbarte Zehenstrecker- oder Peroneus-tertius-Sehne als Transplantat verwendet werden. Postoperativ erfolgt eine Ruhigstellung im Cast für 4 Wochen in Funktionsstellung mit 2-4 Wochen anschließender passiver Beübung.

\section{Extensor-digitorum-longus-Sehne}

\section{Anatomie}

Der M. extensor digitorum longus entspringt der lateralen Tibiakondyle sowie dem Vorderrand der Fibula und der Membrana interossea cruris und wird 


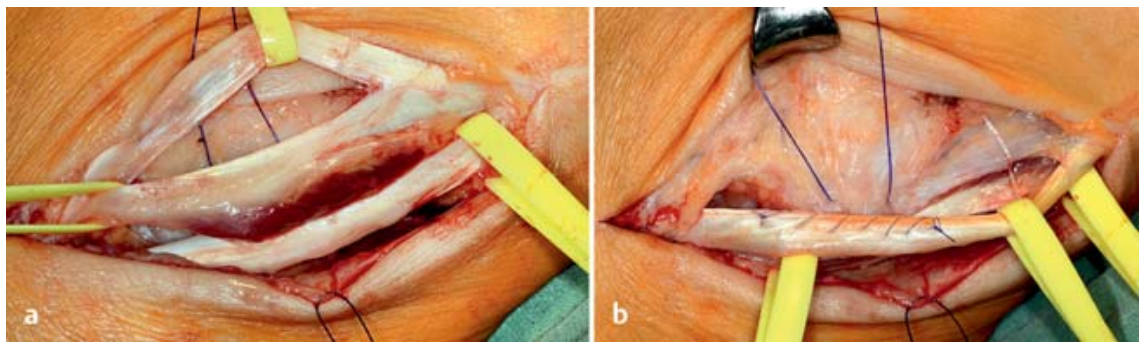

Abb. 4a und b a Längsriss der Peroneus-brevis-Sehne bei chronischer posttraumatischer Instabilität mit rezidivierenden Subluxationsereignissen der Sehne vor die Außenknöchelspitze. b Die Therapie besteht in Débridement und Längsnaht mit Tubulisierung sowie einer Resektion des sehr tiefen Muskelbauchs. Das Retinakulum wird abschließend gerafft und an das Labrum am Fibulahinterrand genäht.

ebenfalls durch den N. peroneus profundus innerviert. Die Sehne teilt sich auf Höhe des oberen Strecksehnenretinakulums in 2 Zügel, die sich wiederum auf Höhe des unteren Strecksehnenretinakulums nochmals teilen. Die 4 Sehnenzügel inserieren an den streckseitigen Endphalangen der Kleinzehen. Neben der Streckung der Kleinzehen bewirkt der M. extensor digitorum longus parallel eine Eversion und Dorsalextension des Fußes.

Verletzungen der Sehne entstehen wie bei der Ruptur der Sehne des M. extensor hallucis longus durch scharfe, herabfallende Gegenstände oder Komplextraumata. Spontanrupturen oder degenerative Rupturen sind in der Literatur nicht beschrieben.

\section{Diagnostik und Therapie}

Eine fehlende aktive Zehenhebung sowie eine Abschwächung der Dorsalextension und Eversion des Fußes im Seitenvergleich spricht für eine Verletzung der M.-extensor-digitorum-longus-Sehne.

Zur Therapie erfolgt die Exploration und ggf. Erweiterung der Wunde mit direkter End-zu-End-Naht der Sehne.

\section{Peronealsehnen}

Anatomie

Der M. peroneus longus entspringt am Kopf und den proximalen 2 Dritteln der Facies lateralis der Fibula, der M. peroneus brevis hat seinen Ursprung am distalen Drittel der Facies lateralis der Fibula. Die beiden Sehnen verlaufen gemeinsam in einer Gleitrinne an der hinteren Fibula und werden dort vom oberen Peronealsehnenretinakulum gehalten. Die Peroneus-brevis-Sehne verläuft anschließend einzeln durch eine Rinne am lateralen Kalkaneus und setzt an der Metatarsale5-Basis an. Die Peroneus-longus-Sehne zieht unterhalb der Peroneus-brevis-Sehne in einer Rinne unter dem Kuboid und hat ihren Ansatz am Os metatarsale I und II sowie dem Os cuneiforme mediale. Neben dem M. peroneus longus und brevis, die vom $\mathrm{N}$. peroneus superficialis innerviert werden, existiert bei ca. $92 \%$ der Bevölkerung ein $M$. peroneus tertius, der als Abspaltung des M. extensor digitorum longus gilt und auch funktionell zu den Fußhebern gehört, sowie ein M. peroneus quartus bei 7-21\%, der als Abspaltung des $M$. peroneus brevis gilt.

Traumatische Rupturen sind sehr selten. Häufiger kommt es zu Partialrupturen bzw. Längsrupturen aufgrund von rezidivierenden Supinationstraumata bzw. einer chronischen (Sub-)Luxation. Hierbei kann das obere Peronealsehnenretinakulum rupturieren und die Sehne(n) vor die Außenknöchelspitze subluxieren bzw. luxieren. Dieses kann akut während eines Supinationstraumas oder einer Kalkaneusluxationsfraktur mit Verschiebung des Tuberfragments nach lateral und kranial oder chronisch mit nicht sicher erinnerlichem Unfall auftreten. Vollständige pathologische Rupturen sind sehr selten und können bei Patienten mit rheumatoider Arthritis auftreten.

\section{Diagnostik}

Klinisch haben die Patienten eine Druckdolenz an der dorsalen Kante der Fibula mit retromalleolärer Schwellung und Funktionsschmerz bei aktiver Pronation und Dorsalextension. Auch kann bei einigen Patienten die spontane Luxation der Peronealsehnen vor den Außenknöchel beobachtet bzw. aktiv ausgelöst werden. MRT und Sonografie erlauben die Beurteilung der Sehnenbeschaffenheit sowie mögliche Engstellen in der Gleitrinne der Fibula durch akzessorische Muskeln. Zusätzlicher Vorteil bei der Sonografie ist die Möglichkeit der dynamischen Untersuchung, bei der das Gleitverhalten und die Subluxation beurteilt werden können. Ein CT sollte vornehmlich bei Sehnenverletzungen bedingt durch knöcherne Engstellen oder Vorsprünge, z.B. bei Problemen nach Kalkaneusfrakturen, erfolgen.

\section{Therapie}

In dem seltenen Fall einer akuten traumatischen Komplettruptur sollte die direkte Sehnennaht nach Osteosynthese der Fraktur erfolgen. Ist diese aufgrund eines $\mathrm{zu}$ ausgeprägten Defekts nicht möglich, kann ein Transfer der Peroneus-longus- auf die Peroneus-brevisSehne erfolgen. Die wesentlich häufiger auftretenden Partialrupturen/Längsrisse der Peroneus-brevis-Sehne hinter dem Außenknöchel werden nach einem lokalen Débridement mit einem feinen monofilen Faden (z.B. 6.0 PDS) fortlaufend genäht (Abb. 4). In gleicher Sitzung muss die verursachende Pathologie behoben werden. Hierzu gehört z.B. die Tenosynovektomie bei Tenosynovitis, die Resektion eines M. peroneus quartus, oder die Resektion eines tief liegenden Bauches des M. peroneus brevis, welche die Führungsrinne zusätzlich verengen können.

In jedem Fall sollte bei akuten und chronischen Luxationen das obere Peronealsehnenretinakulum rekonstruiert werden. Je nach Verletztungstyp erfolgt die Refixierung bei akuter Luxation mittels Direktnaht bzw. intraossären Nähten oder Nahtankern. Bei chronischen Verletzungen wird das ausgewalzte Retinakulum gedoppelt bzw. gerafft, ggf. mit einem Periostlappen verstärkt oder mit einem Nahtanker refixiert. Die konservative Behandlung von Peronealsehnenluxationen im Gips mit 6 Wochen Ruhigstellung ist mit einer hohen Reluxationsrate verbunden.

Neben den beschriebenen Möglichkeiten der Weichteilrekonstruktion gibt es zahlreiche operative Verfahren zur Verbesserung der knöchernen Führung der Peronealsehnen. Diese werden im eigenen Vorgehen zurückhaltend eingesetzt. Die Nachbehandlung erfolgt für 6 Wochen im Unterschenkel-Cast mit beschwerdeadaptierter Belastung in Neutralstellung.

\section{Zusammenfassung}

Die Anzahl der akuten traumatischen Sehnenverletzungen im Vergleich zu degenerativen Veränderungen ist gering. 
Für akute Verletzungen sollte die 1. Wahl immer die direkte End-zu-EndNaht oder Re-Insertion sein. Bei veralteten und degenerativen Sehnenverletzungen ist die direkte Naht in vielen Fällen nicht möglich. Aus diesem Grund sind häufig Ersatzplastiken, Muskeltranspositionen, Korrekturosteotomien oder - in seltenen Fällen - Arthrodesen notwendig. Nicht selten werden Sehnenverletzungen am Fuß bei gleichzeitig vorliegenden Frakturen primär übersehen, weswegen der gründlichen Prüfung der Sehnenfunktion nach der Frakturversorgung eine große Bedeutung zukommt.

\section{Literatur}

${ }^{1}$ Wilhelm K. Neue Aspekte zur Genese der Achillessehnenruptur. Zentralbl Chir 1977; 102: 794-801

2 Anzel SH, Covey KW, Weiner AD et al. Disruption of muscles and tendons; an analysis of 1 , 014 cases. Surgery 1959; 45: 406-414
${ }^{3}$ Zwipp H, Rammelt S. Tscherne Unfallchirurgie Fuß. Berlin, Heidelberg, New York: Springer; 2014

${ }^{4}$ Zwipp H, Dahlen C, Amlang $M$ et al. Verletzungen der Sehne des M. tibialis posterior: Diagnostik und Therapie. Orthopäde 2000; 29 : 251-259

5 Johnson KA, Strom DE. Tibialis posterior tendon dysfunction. Clin Orthop Relat Res 1989; 239: 196-206

${ }^{6}$ Myerson MS, Corrigan J. Treatment of posterior tibial tendon dysfunction with flexor digitorum longus tendon transfer and calcaneal osteotomy. Orthopedics 1996; 19: 383-388

7 Sammarco VJ, Sammarco GJ, Henning C et al. Surgical repair of acute and chronic tibialis anterior tendon ruptures. J Bone Joint Surg Am 2009; 91: 325-332

${ }^{8}$ Franck WM, Olk A, Hennig FF. Combined rupture of the tibialis anterior and the extensor hallucis longus tendons-functional reconstruction. Arch Orthop Trauma Surg 2005; 125: 277-280

${ }^{9}$ Forst R, Forst J, Heller KD. Ipsilateral peroneus brevis tendon grafting in a complicated case of traumatic rupture of tibialis anterior tendon. Foot Ankle Int 1995; 16: 440-444

${ }^{10}$ Maffulli N. Rupture of the Achilles tendon. J Bone Joint Surg Am 1999; 81: 1019-1036
Dr. med Jens Goronzy

Arzt in Weiterbildung

Prof. Dr. med. Stefan Rammelt

Sektionsleiter Sprunggelenk-, Fuß- und

Kinderorthopädie

UniversitätsCentrum für Orthopädie \&

Unfallchirurgie

Universitätsklinikum Carl Gustav Carus

Fetscherstraße 74

01307 Dresden

stefan.rammelt@

uniklinikum-dresden.de 